\title{
Individualized Clinical Target Volume Delineation and Efficacy Analysis in Unilateral Nasopharyngeal Carcinoma Treated with Intensity-Modulated Radiotherapy (IMRT): 10-Year Summary and Results
}

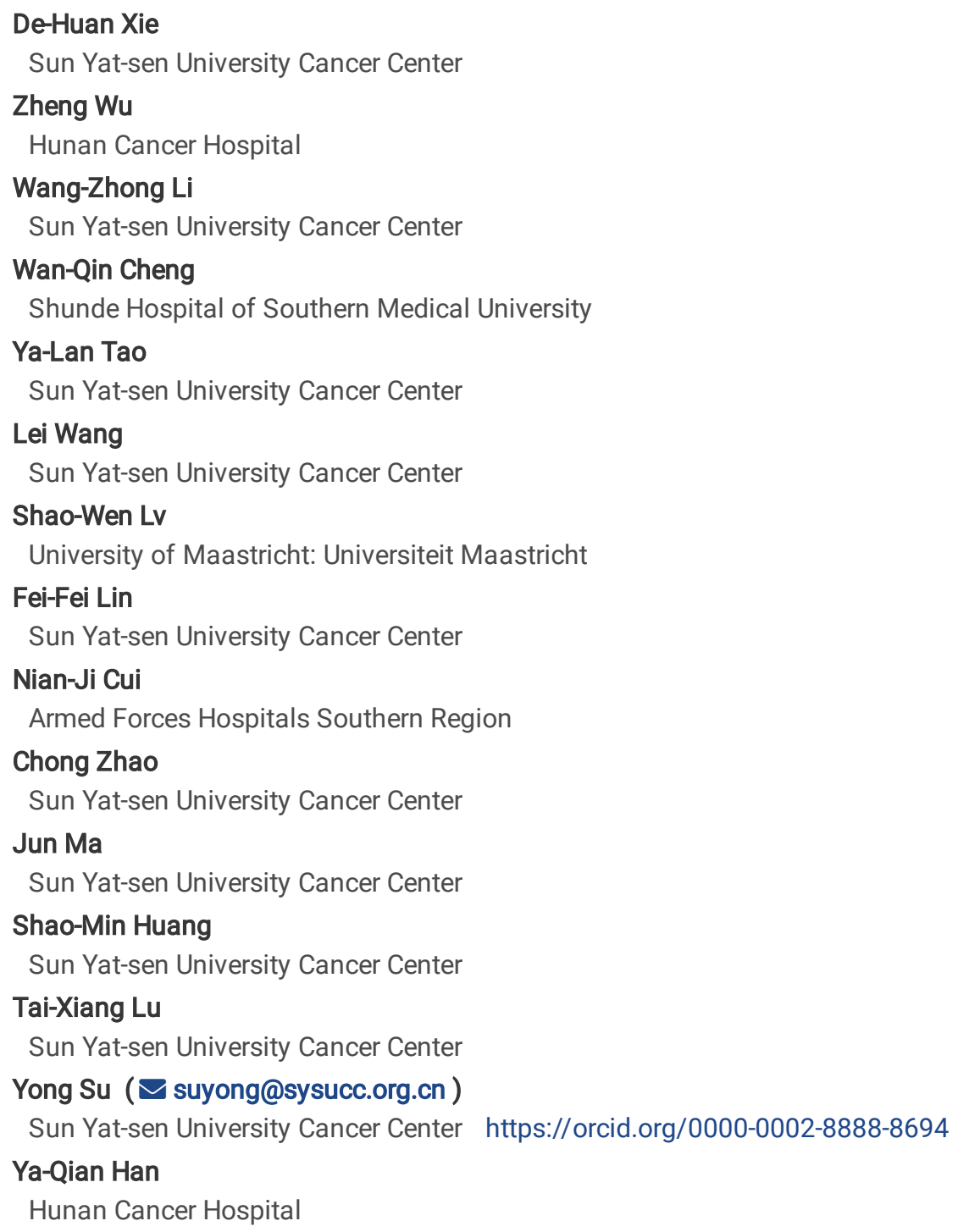




\section{Abstract}

Purpose: To evaluate the long-term local control, failure patterns, and toxicities after individualized clinical target volume (CTV) delineation in unilateral nasopharyngeal carcinoma (NPC) treated with intensity-modulated radiotherapy (IMRT).

Methods: Unilateral NPC was defined as nasopharyngeal mass confined to one side of nasopharynx and did not significantly exceed the midline of nasopharyngeal apex/posterior wall. From November 2003 to December 2017, 95 patients with long-term follow-up were retrospectively included. All patients received IMRT. The CTVs were determined based on the distance from the gross tumor, the contralateral parapharyngeal space and skull base orifices were spared from irradiation.

Results: There were 3 local recurrence and 8 regional recurrences in 10 patients during 84- month follow-up. All local recurrences were PGTVnx-in-field, and no recurrences in traditional high-risk area including contralateral parapharyngeal space and skull base orifices. The 10-year local-recurrence free survival, regional-recurrence free survival and overall survival were $96.2 \%, 90.5 \%$ and $84.7 \%$, respectively. The dosimetry parameters of the tumor-contralateral organs were all lower than the values of the tumor-ipsilateral side $(P<0.05)$. The late toxicities occurred mainly in the tumor-ipsilateral organs, including radiation-induced temporal lobe injury, impaired visuality, hearing loss and subcutaneous fibrosis.

Conclusion: Individualized CTV delineation in unilateral NPC could yield excellent long-term local control with limited out-of-field recurrences, reduced dose to tumor- contralateral organs and mild late toxicities, which is worthy of further exploration.

\section{Introduction}

Intensity-modulated radiation therapy (IMRT) is the standard radiotherapy technique for nasopharyngeal carcinoma (NPC). In the era of IMRT, the prognosis of NPC has significantly improved, mainly due to the markedly improved local control rate (LCR), with the 5-year LCR exceeding $90 \%$ reported by several cancer centers ${ }^{[1-4]}$. In terms of local recurrence patterns, mainly the in-field recurrences were reported in various literatures, mostly commonly the gross tumor volume (GTV) in-field recurrence, and rarely the out-of-field recurrence ${ }^{[1-4]}$. However, it should be noted that although the incidence and severity of xerostomia have significantly decreased under IMRT setting, cured NPC patients still have some radiation-related sequelae, such as hearing loss, radiation-induced temporal lobe injury (TLI), endocrine dysfunction, etc ${ }^{[5,6]}$. Therefore, on the basis of the existing target delineation standards, it is worthy to discuss and try to reduce the irradiation range and radiation-related toxicities or side effects in NPC patients.

In recent 20 years, the target delineation principle of NPC is mainly based on fixed anatomical position, and the prophylactic irradiation range has not fundamentally changed ${ }^{[7,8]}$. The clinical target volume (CTV) range usually needs to include the traditional high-risk areas in the era of conventional radiotherapy, such as the posterior maxillary sinus, the inferior sphenoid sinus, partial clivus, parapharyngeal space, and the skull base orifice. In 2017, specialists on NPC treatment from multiple regions reviewed and discussed the previous clinical and pathological evidence for NPC, and developed an international guideline on target delineation for NPC ${ }^{[9]}$. In this guideline, the $5+5 \mathrm{~mm}$ principle was established, but most experts still recommended that some traditional high-risk areas be prophylactically irradiated regardless of tumor stage.

Scholars have been exploring to optimize the target volume to reduce the long-term adverse effects of NPC patients. In 2019, Sanford et al. ${ }^{[10]}$ proposed an individualized CTV delineation based on tumor invasion trends, without requiring all traditional high-risk areas to be included. Long-term follow-up results showed good efficacy and no out-of-filed recurrence. Since 2003, we have been exploring the individual CTV delineation in NPC with a focus on unilateral NPC population. The principle of CTV delineation was: to set the CTVs based on the distance of the gross tumor, without requiring to include the specific high-risk structures. We then investigated the safety and feasibility of the contralateral parapharyngeal, skull base and even contralateral nasopharyngeal mucosa after sparing from delineation. Here, we report a ten-year exploration and result in this field.

\section{Methods And Materials}

\section{Patient characteristics}

From November 2003 to December 2017, a total of 101 cases of initially treated patients with unilateral NPC were admitted to our research group at the Sun Yat-sen University Cancer Center (SYSUCC), among which 6 cases were lost to follow-up, thus, 95 cases were included in long-term analysis. Unilateral NPC was defined as nasopharyngeal mass confined to one side of nasopharynx and did not significantly exceed the midline of nasopharyngeal apex/posterior wall detected by electronic nasopharynxgoscope (ENS) and magnetic resonance 
imaging (MRI) (Figure 1A). The included criteria for patients were: (1) initially pathologically confirmed unilateral NPC; (2) > 18 years old; (3) treated with IMRT; (4) the performance status (PS) was 0 or 1; (5) no distant metastasis; (6) informed consent related to treatment signed. Exclusion criteria: (1) metachronous or synchronous malignancy; (2) pregnancy or lactation; (3) mental disorders. All patients were staged according to the $7^{\text {th }}$ American Joint Committee on Cancer (AJCC) staging classifications. The study conformed to the ethical guidelines of the Declaration of Helsinki, and the protocol was approved by the Ethics Committee of SYSUCC with the approved number of B2019-16901.

\section{Treatment}

All patients received IMRT. Sixty-three patients received a one-course radiotherapy IMRT and 32 patients received an adaptive replanning $I_{M R T}^{[11]}$. Six, 6 and 8 patients with stage I, II and III diseases, respectively, received radiotherapy alone. Six, 32 and 10 patients with stage II, III and IV diseases, respectively, received concurrent chemoradiotherapy (CCRT). Sixteen and 11 patients with stage III and IV disease, respectively, received induction chemotherapy (IC) plus CCRT.

\section{Radiation therapy}

\section{Radiotherapy positioning}

All patients were immobilized using a head-and-neck thermoplastic mask in a supine position, followed by computed tomography (CT) simulation using 3-mm slices scanned from the top of the head to 2- $\mathrm{cm}$ sub-clavicle. For patients treated with replanning IMRT, a second CT simulation was performed at 22 fractions of radiotherapy.

\section{Definition of target volumes}

The definition of target volumes and organs at risk (OARs) followed the International Commission on Radiation Units and Measurement (ICRU) Reports No. 50 and No. 62. The principles of target volume delineation were referred to the norms of SYSUCC ${ }^{[12]}$ and were revised according to the method applied in our research group ${ }^{[11,13]}$. From November 2003 to May 2013, GTVnx was defined as all gross disease detected by clinical, imaging and endoscopic examination. GTVrpn/nd was defined as the metastatic retropharyngeal lymph nodes/neck lymph nodes detected by clinical and imaging examination; The high-risk CTV (CTV1) was defined as a subclinical disease consisting of $10 \mathrm{~mm}$ margin surrounding GTVnx (not including all nasopharyngeal mucosa); CTVrpn/nd was defined as 0.5-1 cm margin surrounding GTVrpn/nd; The low-risk CTV (CTV2) was defined as a 0.5-1 cm margin surrounding CTV1, including all nasopharyngeal mucosa within contralateral pharyngobasilar fascia, and including the bilateral prophylactically irradiated lymph drainage areas (bilateral retropharyngeal lymph node area, levels Ila, Ilb, III, and Va are routinely covered for all NO patients, whereas ipsilateral levels IV and Vb are also included for N1-3 patients). The illustration of target volume delineation was shown in Figure 1B. From 2013 till now, CTV2 was defined as a 0.5-1 cm margin surrounding CTV1, not including all nasopharyngeal mucosa within contralateral pharyngobasilar fascia, and including the bilateral prophylactically irradiated lymph drainage areas. Besides that, the delineation of other target volumes, including the bilateral prophylactically irradiated lymph drainage areas, remained the same as above mentioned (Figure 1C).

For the delineation of OARs, the temporal lobe, brain stem, spinal cord, optic chiasma, optic nerve, lens, pituitary gland, oral cavity, oropharynx, hypopharynx, parotid gland, submandibular gland, thyroid gland, middle ear, temporomandibular joint and mandible were delineated according to Radiation Therapy Oncology Group (RTOG) 0225 and the practice of our hospital ${ }^{[14]}$. According to the standards of our hospital and the requirements of radiotherapy quality control and quality assurance, planning target volume (PTV) were created by expanding 3-5 $\mathrm{mm}$ from all target volumes from the head-to-foot, front-to-back, and left-to-right directions around the target volumes mentioned above to compensate for geometric uncertainties and patient movements, such as PGTVnx, PGTVrpn/nd, PCTV1, PCTVnd, and PCTV2.

\section{Dose prescription}

From November 2003 to May 2013, the dose prescription of one-course IMRT were: PGTVnx/rpn 68 Gy/30 fractions (F), PGTVnd 62-66 Gy/30 F, PCTV1 60 Gy/30 F and PCTV2 50-54 Gy/30 F. From June 2013 to December 2017, the dose prescription of replanning IMRT were: PGTVnx/rpn 68-69.5 Gy/31-32 F, PGTVnd 68-69.5 Gy/31-32 F, PCTV1 60-61 Gy/31-32 F, PCTV2 45-47 Gy/25-26 F [11]. Dose constraints and evaluation of OARs for patients with one- course IMRT and patients with replanning IMRT were referred to our hospital and RTOG 0225 guidelines and our previous studies ${ }^{[11,13]}$, respectively. Dose constraints for the contralateral unaffected OARs: according to the distance between OARs and the target volumes, stricter constraints were imposed on the basis of the standard limits to reduce dose to the unaffected organs. 


\section{Chemotherapy}

Seventy-five patients received chemotherapy. Among them, 48 patients received concurrent chemotherapy, including cisplatin weekly regimen $\left.(30 \mathrm{mg} / \mathrm{m})^{2}\right), 4-6$ times in total and cisplatin three-week regimen $\left.(80 \mathrm{mg} / \mathrm{m})^{2}\right), 2-3$ times in total. A total of 27 patients received IC combined with concurrent chemotherapy: IC regimen was TPF (docetaxel $60 \mathrm{mg} / \mathrm{m}^{2}$, cisplatin $60 \mathrm{mg} / \mathrm{m}^{2}$, fluorouracil 500 $\mathrm{mg} / \mathrm{m}^{2}$ continuous venous perfusion for $120 \mathrm{~h}$ ), 3-4 times in total; concurrent chemotherapy was a weekly regimen containing platinum, including cisplatin $\left(30 \mathrm{mg} / \mathrm{m}^{2}\right)$, nedaplatin $\left(30 \mathrm{mg} / \mathrm{m}^{2}\right), 4-6$ times in total.

\section{Evaluation of toxicities}

The Common Terminology Criteria for Adverse Event (version 4.0) was used to evaluate treatment-related acute toxicities, and the Late Radiation Morbidity Scoring Criteria of RTOG was used to evaluate radiotherapy-related toxicities. Acute toxicities were defined as those occurring either during the course of IMRT and chemotherapy or within 3 months of its completion, while late toxicities were the toxicities occurred at least 3 months after radiotherapy.

\section{Evaluation and follow-up}

All patients received detailed examinations before and after treatment including medical history, physical examination, hematological examination, chest X-ray, abdominal B-ultrasound, emission computed tomography (ECT) on bone, ENS, and head and neck enhanced MRI or positron emission tomography-computed tomography (PET/CT). Local recurrence was confirmed by endoscopic biopsy and/or MRI. Distant metastases were clinically confirmed by clinical symptoms, physical examination, and imaging examinations and pathological biopsies if necessary. Patients were followed-up once every 3 months within 3 years after radiotherapy, once every 6 months after 3 years, and once every 5 years after 5 years. Whenever possible, salvage treatments (including re-IMRT, surgery, and chemotherapy) were provided for patients who developed relapse or persistent disease.

\section{Survival analysis}

Survival was calculated as the duration from the date of pathological diagnosis to the date of death or the last follow-up. Our primary endpoints were local-recurrence free survival (LRFS) and patterns of local failure, which were classified as "in-field" if $95 \%$ recurrent tumor volume was within the $95 \%$ isodose, "marginal" if 20 - $95 \%$ recurrent tumor volume was within the $95 \%$ isodose or "out-of-field" if $<20 \%$ recurrent tumor volume was within the $95 \%$ isodos $\mathrm{e}^{[15]}$. The local recurrence patterns were determined by re-delineating the tumor volume detected by MRI at the time of recurrence in the original plan evaluation system, and the relationship between the recurrence area and the original dose coverage was analyzed. Our secondary endpoints were overall survival (OS), regional relapse-free survival (RRFS), distant metastasis-free survival (DMFS) and progression-free survival (PFS).

\section{Statistical analysis}

Continuous data followed a normal distribution were analyzed in a t-test, while Wilcoxon rank sum test was used for non-normal distribution data when comparing two groups. LRFS, OS, RRFS, DMFS and PFS were estimated by Kaplan-Meier survival analysis. Statistical analysis and the survival curve were generated by $\mathrm{R}$ Studio version 1.2.1335. Bilateral test $P<0.05$ was considered statistically significant.

\section{Results}

\section{Patient characteristics}

From November 2003 to December 2017, 95 unilateral NPC patients undergoing long-term follow-up were included in the analysis. The median age of the patients was 46 years (21-77 years). Fifty patients with primary tumor located on the right side of nasopharynx, while 45 cases on the left side of nasopharynx. Seventy-seven patients (81.1\%) with stage III-IV, 89.6\% of whom received chemotherapy. Detailed data are shown in Table 1.

\section{Survival results}

As of August 31, 2020, with a median follow-up of 84 months (24-199 months), 3 patients had local recurrence, 7 patients had regional recurrence, 9 patients developed distant metastasis, and a total of 12 patients died ( 3 died of local regional recurrence, 7 died of distant metastasis, 1 died of colon cancer, and 1 died of unknown cause). The 5-year LRFS, RRFS, OS, PFS and DMFS of the whole patients were 97.9\% [95\% confidence interval (Cl): 95.0\%- 100\%), 92.4\% (95\% Cl: 87.1\%- 98\%), 88.1\% (95\% Cl: 81.4\%- 95.4\%), 82.1\% (95\% Cl: $74.4 \%-$ 
90.5\%) and 89.5\% (95\% Cl: 83.1\%- 96.3\%);10-year LRFS, RRFS, OS, PFS and DMFS were $96.2 \%$ (95\% Cl: 91.9\%- 100\%), 90.5\% (95\% Cl: 84.3\%- 97.2\%), 84.7\% (95\% Cl: 77.0\%- 93.3\%), 77.4\% (95\% Cl: 68.8\%- 87.1\%) and 87.8\% (95\% Cl: 80.8\%- 95.3\%) (Figure 2).

\section{Failure patterns}

All local failures were located in the high-dose GTV irradiation area. Among them, 1 patient was staged as T4, with local residue after radiotherapy, progressed 12 months later, and eventually died of local progression (Figure 3A-1 \& A-2). The other 2 patients were all staged as T3. One patient had in situ recurrence of the nasopharyngeal lateral wall 66 months after treatment, and was still free of recurrence and metastasis after re-radiotherapy (Figure 3B-1 \& B-2). The other case developed in situ recurrence at the longus cephalus in tumor-ipsilateral sideand multiple bone metastases 25 months after radiotherapy. After re-radiotherapy and systemic treatment, the patient was still alive with bone metastasis at the end of follow-up and had survived for 49 months since diagnosis (Figure 3C-1 \& C-2).

Among the 7 regional recurrence cases, 1 patient diagnosed as T1N2 had lymph node in lower parotid recurrence determined as out-of-field recurrence. Before treatment, this patient had significant extracapsular invasion in level-II lymph nodes in the tumor relapsed side. One patient diagnosed as T3N3b had PGTVnd in-field recurrence in the level II region in the tumor-ipsilateral side accompanied with contralateral level IV region PCTV2 in-field recurrence. The remaining patients had PGTVnd in-field recurrence. The detailed illustration and description of failure patterns were shown in Table 2.

\section{Dosimetric comparison of OARs}

The dosimetry of the OARs was included in the analysis. The mean dose (Dmean), minimum dose (Dmin) and maximum dose (Dmax) of the tumor-contralateral OARs were all significantly lower than the values of the tumor-ipsilateral side $(P<0.05)$. Furthermore, absolute dose reduction percentage of organs demonstrated a higher dose in tumor-ipsilateral side relative to tumor-contralateral side. See Table $\mathbf{3}$ for details. Additionally, Figure 4 illustrated an advanced-staged patient with the primary tumor locating in the left side pharyngeal recess, showing that the doses to the contralateral temporomandibular joint, mandible (5000 cGy dose line) and contralateral chiasm (3000 cGy dose line) were significantly lower than those to the ipsilateral tumor.

\section{Toxicities}

The most common acute toxicity is Grades I- II reactions. Grade III reactions were mainly oropharyngeal mucositis (15.8\%) and leukopenia (12.6\%), while Grade IV mucositis and Grade IV leukopenia occurred only 1 case, respectively. The detailed acute toxicities are shown in Table 4. By the last follow-up, for late toxicities, 94 patients (98.9\%) had Grade I-II xerostomia. Eight patients (8.4\%) developed radiationinduced TLI on the tumor- ipsilateral side (7 patients with imaging manifestations and 1 patient with clinical symptoms), among which 1 patient suffered radiation-induced TLI on the tumor-unaffected side. One patient and 10 patients had impaired visuality and impaired hearing on the tumor- ipsilateral side, respectively, among which 1 patient had impaired hearing on the tumor-contralateral side. Five patients developed subcutaneous fibrosis after radiotherapy, all of them had cervical lymph node metastasis, among which 1 patient with N2 disease developed bilateral subcutaneous fibrosis.

\section{Discussion}

Unilateral NPC accounts for approximately $10 \%$ of all cases of NPC ${ }^{[16]}$. In the current specification for target volume delineation of NPC, bilateral tissues and structures, such as parapharyngeal space and skull base orifices, are still required to be included in the prophylactic irradiation range for this type of patients ${ }^{[9]}$. However, unilateral NPC is relatively far away from the contralateral parapharyngeal space and contralateral skull base, and jumping invasion is rare. Therefore, it may not be necessary to include contralateral parapharyngeal structures and skull base orifices in the target volume. Therefore, we explored the feasibility of sparing contralateral parapharyngeal space and skull base orifices from irradiation. The long-term follow-up results of this group showed that the 10-year results were achieved $96.2 \%, 90.5 \%$ and $84.7 \%$ for LRFS, RRFS and OS respectively, reaching a satisfied efficacy.

For unilateral NPC, individualized CTV delineation method was adopted. In this study, the CTV range was set according to the distance from gross tumor based on the experience of surgical resection margin for squamous cell carcinoma of head and neck (HNSCC), and all traditional high-risk structures were not required to be included. From 2003 to 2013, CTV1 was set as a $1 \mathrm{~cm}$ margin surrounding GTVnx and CTV2 a 0.5-1 mm margin surrounding CTV1, but all nasopharyngeal mucosa needed to be included. After 10 years of exploration, there was no recurrence of contralateral parapharyngeal structure. After 2013, we further set CTV2 as a 0.5-1 cm margin surrounding CTV1, without even requiring to include all contralateral nasopharyngeal mucosa. After further narrowing the CTV, no recurrence in contralateral structures and contralateral unirradiated nasopharyngeal mucosa was observed. An individual CTV delineation study from Harvard 
Medical School did not require to include the contralateral parapharyngeal space in CTV for unilateral NPC, and the long-term follow-up showed no recurrence in the contralateral parapharyngeal space ${ }^{[10]}$.

In the present study, the determined irradiation range was about $10 \mathrm{~mm}+5 \mathrm{~mm}$ from the gross tumor, but could it be further reduced on this basis? The CTV has been delineated according to the principle of $5+5 \mathrm{~mm}$ in HNSCC [17], and this principal was also recommended in the international guideline for NPC, ${ }^{[9]}$. However, in the future CTV delineation, whether we can completely learn from the experience of HNSCC and only delineate $5+5 \mathrm{~mm}$ in NPC without including specific high-risk structures is worthy of further exploration and attempt. Surgical resection is an important treatment method for locally recurrent NPC patients. For patients who can be surgically resected, the resection range is generally $0.5-1 \mathrm{~cm}$ around the gross tumor ${ }^{[18]}$. Therefore, this also provides a basis and reference for our future CTV optimization of NPC.

It should be noted that the contralateral structures would still receive a certain irradiation dose even though the contralateral structures were not delineated in the CTV under IMRT technology. Meanwhile, routine prophylactic retropharyngeal region irradiation was required regardless of retropharyngeal lymph node metastasis ${ }^{[9]}$. This could also lead to a dose of exposure to nearby traditional high-risk areas. However, this is mainly due to the limitations of existing IMRT technology. Moreover, the scattering doses to adjacent structures were obviously lower than the values to those delineated in the CTV (Figure 4). Dosimetry analysis also showed that dose to OARs on the tumorcontralateral side was significantly lower than those on the tumor-ipsilateral side with the average Dmean reduction percentage of $7.18 \%$ to $33.02 \%$ of the above-mentioned organs. Long-term follow-up result showed that the incidence of late toxicities on the tumor- contralateral side were also lower than those on the tumor- ipsilateral side, with only 1 patient had hearing loss and radiation-induced TLI on the tumorcontralateral side, respectively.

Our study also has some limitations. Firstly, it was a single sample retrospective study without a control group to compare efficacy and toxicity. Secondly, a long- time span with CTV range constantly optimized under the accumulation of experience, which means not all patients had the same irradiation range. However, we always followed a uniform principle of determining CTV range based on the distance from gross tumor and not necessarily including specific high-risk structures. Thirdly, treatment heterogeneity including the introduction of IC and replanning IMRT, but different treatment regimen has no effect on the irradiation range. Finally, deficiencies in the long-term toxicity evaluation methods due to the retrospective collection of data, making it difficult to fully reflect the quality-of-life benefits brought by the CTV reduction in this study.

In conclusion, our invasion distance- based CTV delineation is feasible for unilateral NPC based on ten-year experience. We did not observe failure in the reduced-irradiated area. By narrowing the irradiation volume and sparing the contralateral parapharyngeal space, even nasopharyngeal mucosal from irradiation, dose to the contralateral normal tissues significantly reduced, thereby reducing the toxicities. This method deserves to be verified and explored by more scholars to make up for the deficiencies of this study.

\section{Abbreviations}

IMRT: Intensity-modulated Radiotherapy

NPC: nasopharyngeal carcinoma

LCR: local control rate

GTV: gross tumor volume

CTV: clinical target volume

TLI: temporal lobe injury

SYSUCC: Sun Yat-sen University Cancer Center

ENS: electronic nasopharynxgoscope

MRI: magnetic resonance imaging

PS: performance status

AJCC: American Joint Committee on Cancer 
CCRT: Concurrent chemoradiotherapy

IC: induction chemotherapy

CT: computed tomography

OARs: organs at risk

ICRU: the International Commission on Radiation Units and Measurement

CTV1: high-risk clinical target volume

CTV2: low-risk clinical target volume

RTOG: Radiation Therapy Oncology Group

PTV: planning target volume

ECT: emission computed tomography

PET/CT: positron emission tomography-computed tomography

LRFS: local-recurrence free survival

OS: overall survival

RRFS: regional relapse-free survival

DMFS: distant metastasis-free survival

PFS: progression-free survival

Dmean: mean dose

Dmin: minimum dose

Dmax: maximum dose

\section{Declarations}

\section{Funding}

The authors declare that they had no funding for this study.

\section{Conflicts of interest/Competing interests}

The authors declare that they have no competing interests.

\section{Availability of data and material}

The datasets used and/or analyzed during the current study were uploaded onto the Research Data Deposit public platform (www.researchdata.org.cn), with the RDD approval number RDDB2020001032.

\section{Authors' contributions}

D.H.X., Z.W., W.Z.L., Y.S., and Y.Q.H. designed the study. D.H.X., Z.W., Y.S., T.X.L., J.M., C.Z., N.J.C., W.Q.C., L.W., and S.W.L. delineated the targets and performed radiation treatment. D.H.X., Z.W., W.Z.L., W.Q.C., Y.L.T., L.W., S.W.L., F.F.L., and S.M.H acquired data. D.H.X., Z.W., W.Z.L., analyzed and interpreted the data. D.H.X., Z.W., W.Z.L., and Y.S. wrote the manuscript, and revised the manuscript. All authors have reviewed the manuscript and approved the final version.

\section{Ethics approval}


Retrospective ethical approval was obtained from the institutional ethics committee of the Sun Yat-sen University Cancer Center, with the approval number B2019-169-01. Written informed treatment consents were required from all patients with respect to chemotherapy and/or radiotherapy.

\section{Consent for publication}

Not applicable.

\section{Acknowledgments}

We thank International Science Editing (http://www.internationalscienceediting.com) for editing this manuscript.

\section{References}

1. Chen Lei,Zhang Yuan,Lai Shu-Zhen et al. 10-Year Results of Therapeutic Ratio by Intensity-Modulated Radiotherapy Versus TwoDimensional Radiotherapy in Patients with Nasopharyngeal Carcinoma.[J] .Oncologist, 2019, 24: e38-e45.

2. Wu Zheng,Wang Lei,Xie De-Huan et al. Failure patterns and prognostic factors for cervical node-negative nasopharyngeal carcinoma in the intensity-modulated radiotherapy era.[J] .Asia Pac J Clin Oncol, 2021, 17: 330-337.

3. Wu Li-Rong,Liu Ya-Tian,Jiang Ning et al. Ten-year survival outcomes for patients with nasopharyngeal carcinoma receiving intensitymodulated radiotherapy: An analysis of 614 patients from a single center.[J] .Oral Oncol, 2017, 69: 26-32.

4. Au K H,Ngan Roger K C,Ng Alice W Y et al. Treatment outcomes of nasopharyngeal carcinoma in modern era after intensity modulated radiotherapy (IMRT) in Hong Kong: A report of 3328 patients (HKNPCSG 1301 study).[J] .Oral Oncol, 2018, 77: 16-21.

5. McDowell Lachlan J,Rock Kathy,Xu Wei et al. Long-Term Late Toxicity, Quality of Life, and Emotional Distress in Patients With Nasopharyngeal Carcinoma Treated With Intensity Modulated Radiation Therapy.[J]. Int J Radiat Oncol Biol Phys, 2018, 102: $340-352$.

6. Zeng Lei,Huang Shao-Min,Tian Yun-Ming et al. Normal Tissue Complication Probability Model for Radiation-induced Temporal Lobe Injury after Intensity-modulated Radiation Therapy for Nasopharyngeal Carcinoma.[J] .Radiology, 2015, 276: 243-9.

7. Lai Shu-Zhen,Li Wen-Fei,Chen Lei et al. How does intensity-modulated radiotherapy versus conventional two-dimensional radiotherapy influence the treatment results in nasopharyngeal carcinoma patients? [J] .Int J Radiat Oncol Biol Phys, 2011, 80: 661-8.

8. Lee Nancy, Xia Ping, Quivey Jeanne M et al. Intensity-modulated radiotherapy in the treatment of nasopharyngeal carcinoma: an update of the UCSF experience. Int J Radiat Oncol Biol Phys, 2002, 53: 12-22.

9. Lee Anne W, Ng Wai Tong, Pan Jian Ji et al. International guideline for the delineation of the clinical target volumes (CTV) for nasopharyngeal carcinoma. Radiother Oncol, 2018, 126: 25-36.

10. Sanford Nina N, Lau Jackson, Lam Miranda B et al. Individualization of clinical target volume delineation based on stepwise spread of nasopharyngeal carcinoma: Outcome of more than a decade of clinical experience. Int J Radiat Oncol Biol Phys, 2019, 103: 654-668.

11. Xie Dehuan, Cheng Wanqin, Lv Shaowen et al. Target delineation and dose prescription of adaptive replanning intensity-modulated radiotherapy for nasopharyngeal carcinoma. Cancer Commun (Lond), 2019, 39: 18.

12. Miao Jingjing,Di Muping,Chen Boyu et al. A Prospective 10-Year Observational Study of Reduction of Radiation Therapy Clinical Target Volume and Dose in Early-Stage Nasopharyngeal Carcinoma.[J] .Int J Radiat Oncol Biol Phys, 2020, 107: 672-682.

13. Wang Lei, Wu Zheng, Xie Dehuan et al. Reduction of target volume and the corresponding dose for the tumor regression field after induction chemotherapy in locoregionally advanced nasopharyngeal carcinoma. Cancer Res Treat, 2019, 51: 685-695.

14. Sun Ying, Yu Xiao-Li, Luo Wei et al. Recommendation for a contouring method and atlas of organs at risk in nasopharyngeal carcinoma patients receiving intensity-modulated radiotherapy. Radiother Oncol, 2014, 110: 390-7.

15. Chao K S Clifford,Ozyigit Gokhan,Tran Binh N et al. Patterns of failure in patients receiving definitive and postoperative IMRT for headand-neck cancer.[J] .Int J Radiat Oncol Biol Phys, 2003, 55: 312-21.

16. Ying Sun, Yu Xiao-Li, Zhang Guang-Shun, et al. Reduction of clinical target volume in patients with lateralized cancer of the nasopharynx and without contralateral lymph node metastasis receiving intensity-modulated radiotherapy[J]. Head \& Neck, 2016, 38(S1): E468-E472.

17. Grégoire Vincent, Evans Mererid, Le Quynh-Thu et al. Delineation of the primary tumour Clinical Target Volumes (CTV-P) in laryngeal, hypopharyngeal, oropharyngeal and oral cavity squamous cell carcinoma: AIRO, CACA, DAHANCA, EORTC, GEORCC, GORTEC, HKNPCSG, HNCIG, IAG-KHT, LPRHHT, NCIC CTG, NCRI, NRG Oncology, PHNS, SBRT, SOMERA, SRO, SSHNO, TROG consensus guidelines. Radiother Oncol, 2018, 126: 3-24. 
18. You-Ping Liu, Wen Yi-Hui, Tang Jun, et al. Endoscopic surgery compared with intensity-modulated radiotherapy in resectable locally recurrent nasopharyngeal carcinoma: a multicentre, open-label, randomised, controlled, phase 3 trial[J]. The lancet oncology, 2021, 22(3): 381-390.

\section{Tables}

Table 1. Patient characteristics

\begin{tabular}{|c|c|}
\hline Characteristic & $\mathrm{N}(\%)$ \\
\hline \multicolumn{2}{|l|}{ Sex } \\
\hline Male & $70(73.7)$ \\
\hline Female & $25(26.3)$ \\
\hline Median age (years old) & 46囚21-77》 \\
\hline \multicolumn{2}{|l|}{ Pathology } \\
\hline Undifferentiated non-keratinized carcinoma & $92(96.8)$ \\
\hline Differentiated non-keratinized carcinoma & $3(3.2)$ \\
\hline \multicolumn{2}{|l|}{ Site } \\
\hline Right & $50 \rrbracket 52.6 \rrbracket$ \\
\hline Left & $45 \llbracket 47.4 \rrbracket$ \\
\hline \multicolumn{2}{|l|}{ T classification } \\
\hline $\mathrm{T} 1$ & $31(32.6)$ \\
\hline $\mathrm{T} 2$ & $14(14.7)$ \\
\hline T3 & $39(41.1)$ \\
\hline $\mathrm{T} 4$ & $11(11.6)$ \\
\hline \multicolumn{2}{|l|}{$\mathrm{N}$ classification } \\
\hline No & $9(9.5)$ \\
\hline $\mathrm{N} 1$ & $41(43.2)$ \\
\hline N2 & $32(33.7)$ \\
\hline N3 & 13(13.7) \\
\hline \multicolumn{2}{|l|}{ Clinic stage } \\
\hline I & $6(6.3)$ \\
\hline II & $12(12.6)$ \\
\hline III & $56(58.9)$ \\
\hline IVa-b & $21(22.2)$ \\
\hline \multicolumn{2}{|l|}{ Treatment } \\
\hline Radiotherapy alone & $20(21.1)$ \\
\hline Induction \& Concurrent-radiotherapy & $27(28.4)$ \\
\hline Concurrent-radiotherapy & $48(50.5)$ \\
\hline \multicolumn{2}{|l|}{ Radiotherapy } \\
\hline One-course IMRT (2003 - 2013) & $63 \rrbracket 66.3 \rrbracket$ \\
\hline Replanned IMRT (2013 - 2017) & 32ه33.7ه \\
\hline
\end{tabular}


Table 2. Analysis of local and regional recurrence

\begin{tabular}{|clllll|}
\hline No. & Stage, $7^{\text {th }}$ & Site of recurrence & Location of recurrence & Actual dose (Gy) & Type of recurrence \\
\hline 1 & T3N0 & NP & PGTV & 68 & PGTV-in-field \\
\hline 2 & T4N2 & NP & PGTV & 66.5 & PGTV-in-field \\
\hline 3 & T3N1 & NP & PGTV & 69 & PGTV-in-field \\
\hline 4 & T1N1 & Ipsilateral RPLN & PGTVrpn & 71.5 & PGTVrpn-in-field \\
\hline 5 & T1N2 & Ipsilateral parotid lymph node & Out of targets & 53 & Out-of-field \\
\hline 6 & T3N2 & Ipsilateral Ib & PGTVnd & 68 & PGTVnd-in-field \\
\hline 7 & T3N3b & Ipsilateral Ilb & PGTVnd & 68 & PGTVnd-in-field \\
\hline 8 & T3N3b & Ipsilateral IV \& & PCTV2 & 50 & PCTV2-in-field \\
\hline 9 & T2N3b & Ipsilateral Ila & PGTVnd & 67 & PGTVnd-in-field \\
\hline 10 & T1N3b & Ipsilateral Vb & PGTVnd & 68 & PGTVnd-in-field \\
\hline
\end{tabular}

Note: NP, nasopharynx; RPLN, retropharyngeal lymph node.

Table 3. Dosimetric comparison of paired normal organs in the tumor- affected side and tumor-non-affected side

\begin{tabular}{|c|c|c|c|c|c|c|c|c|c|c|c|c|}
\hline \multirow[t]{2}{*}{ Organs } & \multicolumn{4}{|c|}{ Average Dmean $₫ \mathrm{~Gy} \rrbracket$} & \multicolumn{4}{|c|}{ Average Dmin $₫ G y \rrbracket$} & \multicolumn{4}{|c|}{ Average Dmax $\llbracket \mathrm{Gy} \rrbracket$} \\
\hline & NA & $A$ & $\Delta$ Dmean $\%$ & $P$ & NA & $A$ & $\Delta \mathrm{Dmin} \%$ & $P$ & NA & A & $\Delta \mathrm{Dmax} \%$ & $P$ \\
\hline Parotid & 33.55 & 39.17 & 13.30 & 0.009 & 13.37 & 17.40 & 18.04 & $<0.001$ & 63.68 & 68.51 & 7.46 & $<0.001$ \\
\hline Mid ear & 24.07 & 35.06 & 27.53 & $<0.001$ & 13.60 & 17.58 & 14.56 & $<0.001$ & 42.00 & 58.44 & 26.85 & $<0.001$ \\
\hline $\begin{array}{l}\text { Temporal } \\
\text { lobe }\end{array}$ & 10.93 & 15.88 & 27.94 & $<0.001$ & 1.27 & 4.54 & 13.02 & $<0.001$ & 51.14 & 67.68 & 23.36 & $<0.001$ \\
\hline TMJ & 27.43 & 43.42 & 33.02 & $<0.001$ & 21.36 & 30.51 & 25.71 & $<0.001$ & 36.97 & 60.45 & 35.53 & $<0.001$ \\
\hline Optic nerve & 16.71 & 24.82 & 26.05 & $<0.001$ & 9.05 & 11.93 & 13.41 & $<0.001$ & 27.47 & 40.35 & 28.09 & $<0.001$ \\
\hline Eye & 7.04 & 8.11 & 8.26 & $<0.001$ & 2.39 & 2.51 & 4.36 & $<0.001$ & 19.65 & 24.93 & 15.51 & $<0.001$ \\
\hline Mandibular & 31.51 & 37.02 & 14.51 & $<0.001$ & 12.90 & 14.06 & 4.90 & $<0.001$ & 60.10 & 66.17 & 9.84 & $<0.001$ \\
\hline SMG & 47.99 & 53.29 & 7.18 & $<0.001$ & 32.66 & 37.94 & 6.17 & $<0.001$ & 62.47 & 66.45 & 5.71 & $<0.001$ \\
\hline
\end{tabular}

Note: TMJ, temporomandibular joint; SMG, submandibular gland; NA: tumor non-affected side; A: tumor affected side; $\triangle \mathrm{Dmean} \% /$ $\Delta \mathrm{Dmin} \% / \Delta \mathrm{Dmax} \%$ was calculated as (Dmean/Dmin/Dmax (tumor ipsilateral side) $^{-}$Dmean/Dmin/Dmax (tumor contralateral side) / Dmean/Dmin/Dmax (tumor ipsilateral side) $\times 100 \%$

Table 4. Incidence of several acute toxicities regarding 0-IV grade 


\begin{tabular}{|c|c|c|c|c|c|}
\hline Grade & 0 & I & II & III & IV \\
\hline Dermatitis & $0 \varangle 0.0 \% \rrbracket$ & 81囚85.2\%区 & 13凶13.7\%区 & $1 \otimes 1.1 \% \bigotimes$ & $0 \otimes 0.0 \% \rrbracket$ \\
\hline Mucositis & $2 \varangle 2.1 \% \rrbracket$ & $54 \bigotimes 56.8 \% \rrbracket$ & $23 \bowtie 24.2 \% \bigotimes$ & $15 \rrbracket 15.8 \% \rrbracket$ & $1 \otimes 1.1 \% \bigotimes$ \\
\hline Nausea & $57 ه 60.0 \% \bigotimes$ & $29 \bowtie 30.5 \% \rrbracket$ & $8 \bowtie 8.4 \% \rrbracket$ & 1ه1.1\%ه & $0 \otimes 0.0 \% \rrbracket$ \\
\hline Visual loss & $94 \otimes 98.9 \% \bigotimes$ & $1 \otimes 1.1 \% \bigotimes$ & $0 \rrbracket 0.0 \% \rrbracket$ & $0 \bigotimes 0.0 \% \bigotimes$ & $0 \otimes 0.0 \% \rrbracket$ \\
\hline Hearing loss & 85囚89.5\%》 & 10冈10.5\%区 & $0 \otimes 0.0 \% \rrbracket$ & $0 \otimes 0.0 \% \bigotimes$ & $0 \otimes 0.0 \% \rrbracket$ \\
\hline Trismus & 95囚100\%》 & $0 \otimes 0.0 \% \rrbracket$ & $0 \otimes 0.0 \% \rrbracket$ & $0 \otimes 0.0 \% \rrbracket$ & $0 \rrbracket 0.0 \% \rrbracket$ \\
\hline Xerostomia & 7区7.4\%区 & $65 \rrbracket 68.4 \% \rrbracket$ & $22 \rrbracket 23.2 \% \bigotimes$ & $1 \otimes 1.1 \% \bigotimes$ & $0 \otimes 0.0 \% \rrbracket$ \\
\hline WBC & $28 \bowtie 29.5 \% \rrbracket$ & $26 \rrbracket 27.4 \% \rrbracket$ & $28 \bowtie 29.5 \% \rrbracket$ & $12 \rrbracket 12.6 \% \rrbracket$ & $1 \otimes 1.1 \% \bigotimes$ \\
\hline PLT & $76 \otimes 80.0 \% \bigotimes$ & $8 \bowtie 8.4 \% \bigotimes$ & $8 \rrbracket 8.4 \% \rrbracket$ & $3 \varangle 3.2 \% \bigotimes$ & $0 \otimes 0.0 \% \rrbracket$ \\
\hline HGB & $78 \rrbracket 2.1 \% \rrbracket$ & $13 \bigotimes 13.7 \% \rrbracket$ & $3 \rrbracket 3.2 \% \rrbracket$ & $1 \otimes 1.1 \% \bigotimes$ & $0 \otimes 0.0 \% \rrbracket$ \\
\hline
\end{tabular}

Note: WBC, white blood cells; PLT, platelet; HGB, hemoglobin.

\section{Figures}

\section{Figure 1}

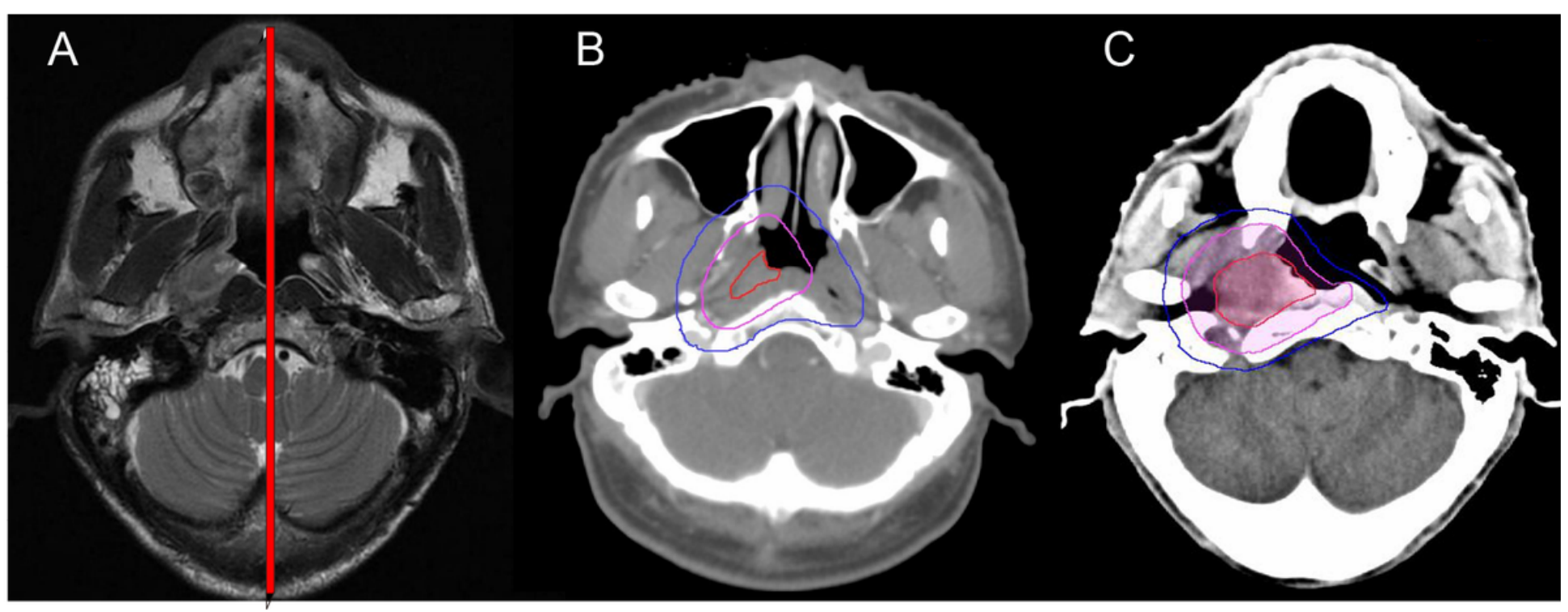

\section{Figure 1}

(A) Representative lateral NPC. (B \& C) Illustrative target delineation of lateral NPC: (B) CTV2 that included all mucosa of nasopharynx; (C) CTV2 that did not include all mucosa of nasopharynx. Red line: GTVnx, pink line: CTV1, blue line: CTV2. 
Figure 2

A

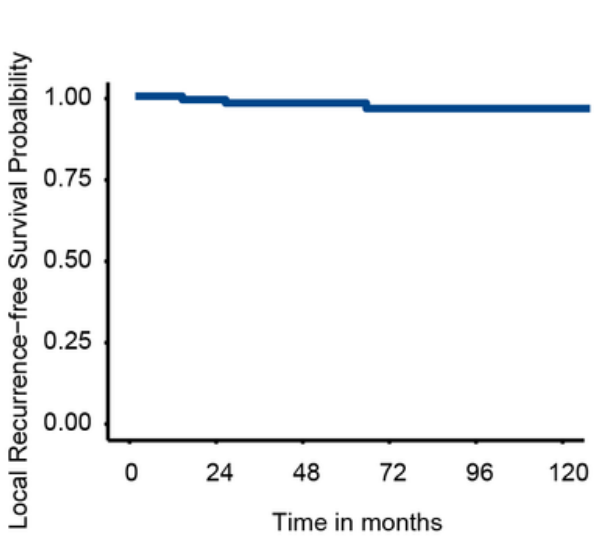

Number at risk

$\begin{array}{llllll}95 & 94 & 73 & 53 & 41 & 21\end{array}$

D

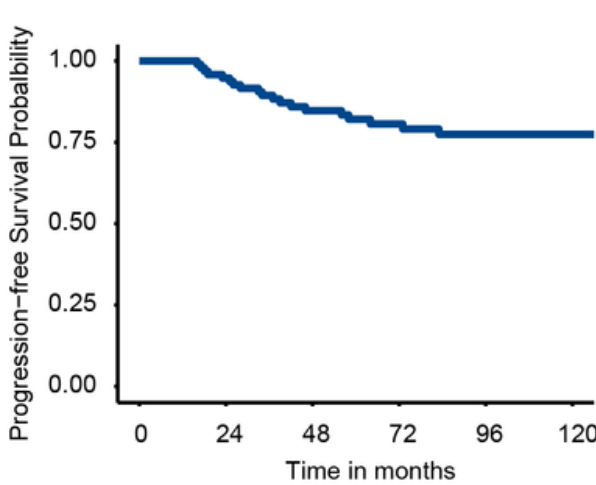

Number at risk

$\begin{array}{rrrrrr}-\quad 95 & 90 & 69 & 52 & 41 & 21\end{array}$
B

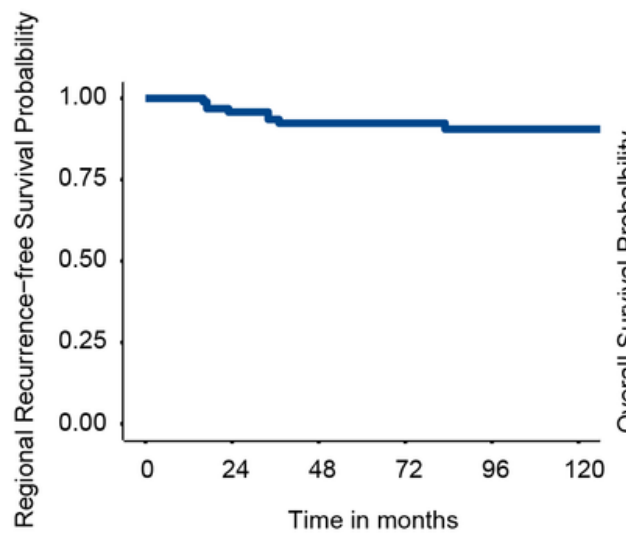

Number at risk

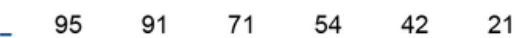

C

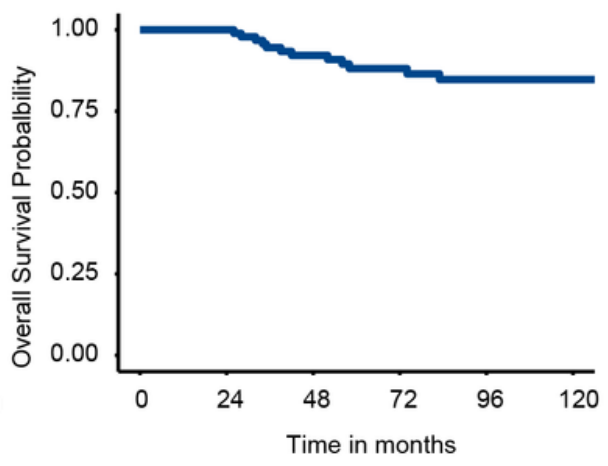

Number at risk

$\mathrm{E}$

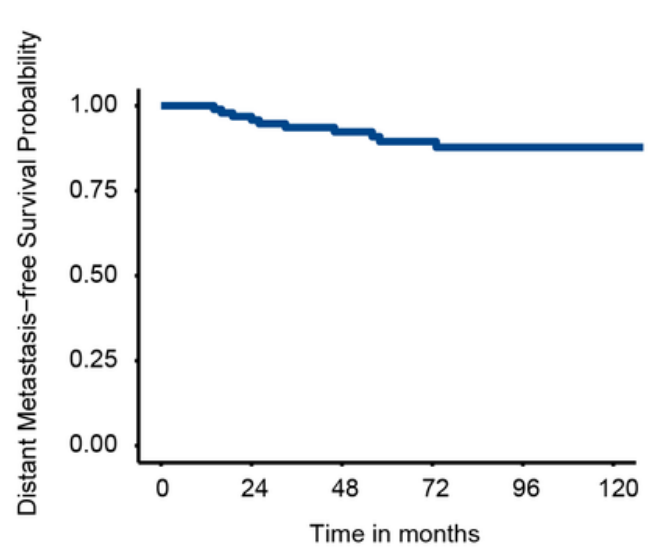

Number at risk

$\begin{array}{rrrrrr}95 & 92 & 72 & 53 & 42\end{array}$

\section{Figure 2}

Survival curves of local relapse-free survival (LRFS), regional relapse-free survival (RRFS), overall survival (OS), progression-free survival (PFS) and distant metastasis-free survival (DMFS). 
Figure 3
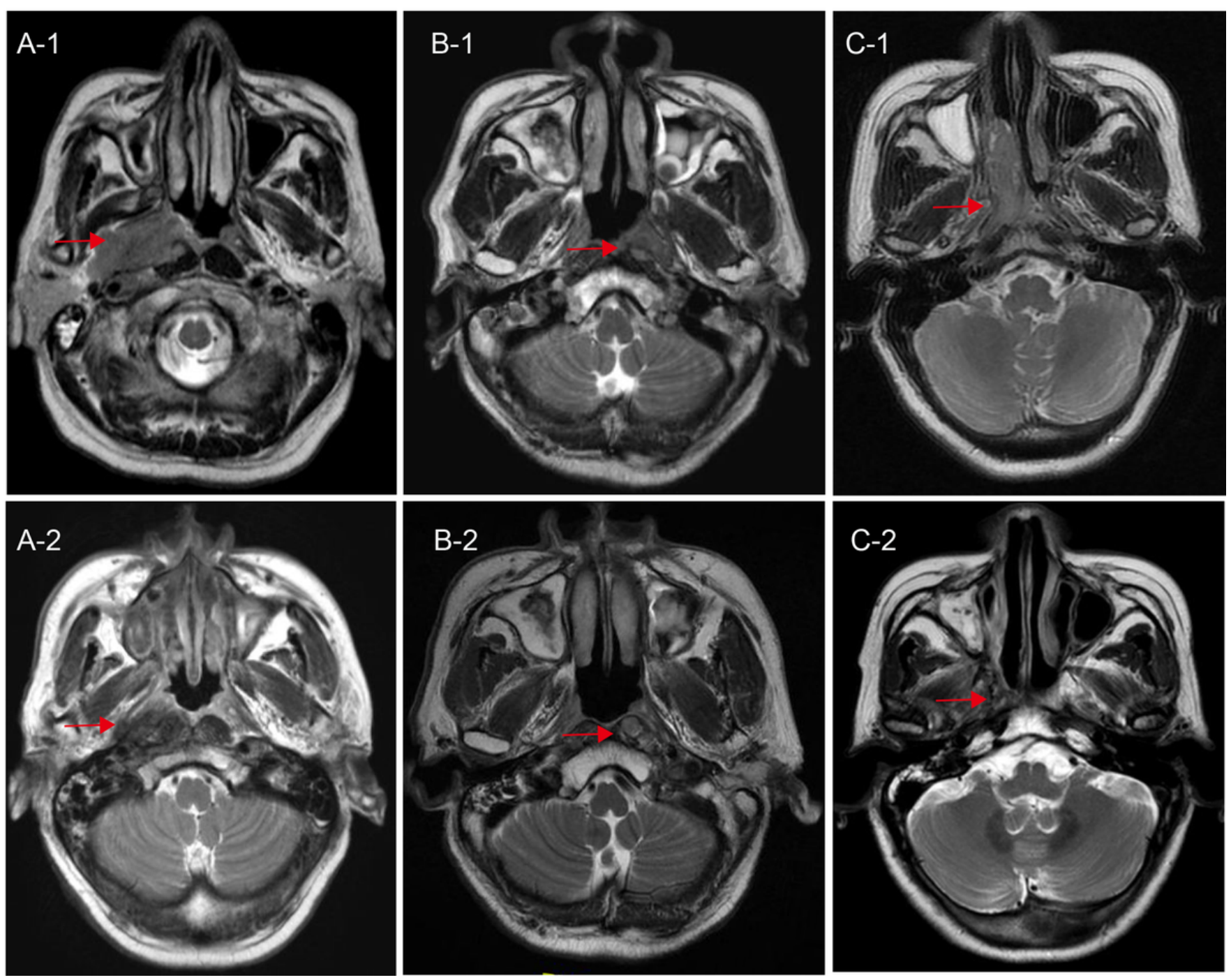

Figure 3

Local recurrence illustration. A-1, B-1 and C-1: MRI showing primary tumor before treatment. A-2, B-2 and C-2: MRI showing corresponding recurred tumor. Red arrow: primary tumor and recurred tumor site. 


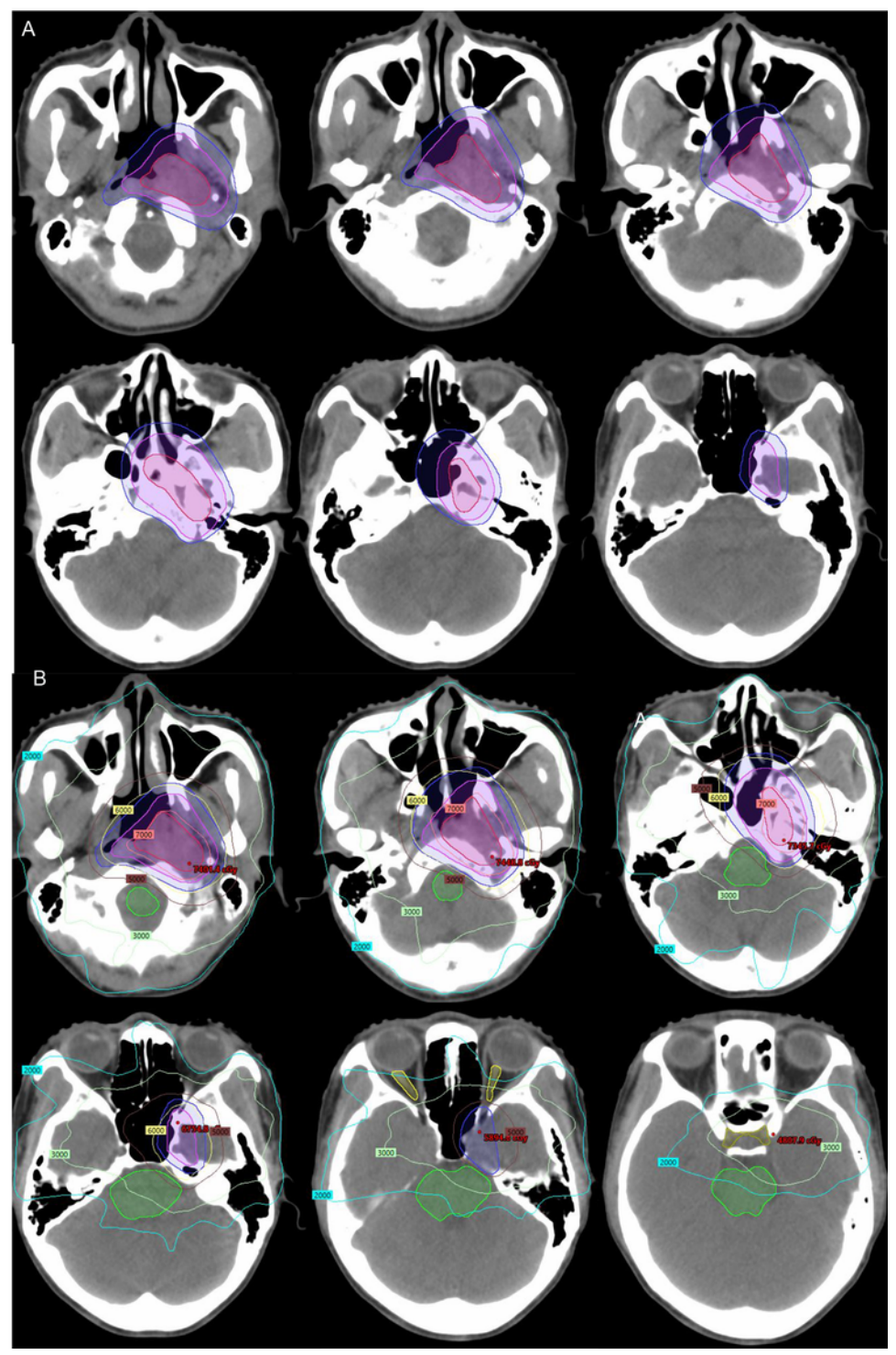

\section{Figure 4}

Target delineation and isodose curve of an advanced-staged NPC patient with the primary tumor locating in the left side pharyngeal recess. As shown in the figure, when the CTV did not delineate the contralateral nasopharyngeal mucosa and parapharyngeal structures, the doses to the contralateral temporomandibular joint, mandible (5000 cGy dose line) and contralateral chiasm (3000 cGy dose line) were significantly lower than those to the ipsilateral tumor. A: target delineation of GTVnx CTV1 and CTV2. B: showing isodose lines. Red line: GTVnx, pink line: CTV1, blue line: CTV2. 